\title{
Observation of enhanced energy transfer in individual quantum dot-oligophenylene vinylene nanostructures
}

\author{
Michael Y. Odoi, ${ }^{1}$ Nathan I. Hammer, ${ }^{1}$ Kevin Sill, ${ }^{2}$ Todd Emrick, ${ }^{2}$ and Michael D. Barnes ${ }^{1}$ \\ ${ }^{1}$ Department of Chemistry, University of Massachusetts, Amherst, MA 01003-9336 \\ ${ }^{2}$ Department of Polymer Science and Engineering, University of Massachusetts, Amherst, MA 01003-9336
}

\section{Preparation of CdSe-oligo(phenylene vinylene) composite nanostructures.}

In an inert atmosphere glovebox, a THF solution of phenybromide-functionalized CdSe nanoparticles (10 $\mathrm{mg} / \mathrm{mL})$ was combined in a glass tube with $\mathrm{N}$-methyldicyclohexylamine $\left(\begin{array}{lll}0.10 & \mathrm{~g}\end{array}\right)$, tetrakis(triphenylphosphine) palladium $(2 \mathrm{mg})$, and 1-bromo-2,5-di- $n$-octyl-4-vinylbenzene $(0.15 \mathrm{~g})$. The tube was sealed with a Teflon valve, removed from the glovebox, and heated to $90{ }^{\circ} \mathrm{C}$ for $16 \mathrm{~h}$. The solution was allowed to cool to room temperature, then centrifuged for 30 minutes. The supernatant was decanted, and the $\mathrm{CdSe}$-oligo(phenylene vinylene) composite material was precipitated using methanol, isolated by centrifugation, dried under a stream of nitrogen, and stored as a solution in THF. ${ }^{1} \mathrm{H}$ NMR spectroscopy on the composite material was used to confirm the successful preparation of oligo(phenylene vinylene): ${ }^{1} \mathrm{H}$ NMR (300 MHz, $\left.\mathrm{CD}_{2} \mathrm{Cl}_{2}, \delta\right) 7.37$ (Ar- $\left.\mathrm{H}\right), 7.27$ (Ar-CH=CH-Ar), $2.58\left(\mathrm{Ar}-\mathrm{CH}_{2}\right), 1.68\left(\mathrm{Ar}-\mathrm{CH}_{2} \mathrm{CH}_{2}\right), 1.21$ (alkyl $\left.\mathrm{CH}_{2}\right), 0.88\left(\mathrm{CH}_{2}-\mathrm{CH}_{3}\right)$ ppm. Photophysical characterization of the composite is described in the text.

\section{Atomic Force Microscope (AFM) Measurements}

The fluorescence spectra and intensity profiles of individual CdSe-OPV nanostructures were correlated directly with particle size, using a Digital Instruments Bioscope Atomic Force Microscope in tapping mode. The measured size distribution $(9-$ $14 \mathrm{~nm}$ ) agrees well with a $4 \mathrm{~nm}$ dot fully decorated with oligo-phenylene vinylene with an average ligand length of 6 monomer units. Figure 1 shows a representative fluorescence image (left), along with a surface height scan of the same area using AFM (right). A z-scan of the particle indicated by the circle is shown in the lower panel.

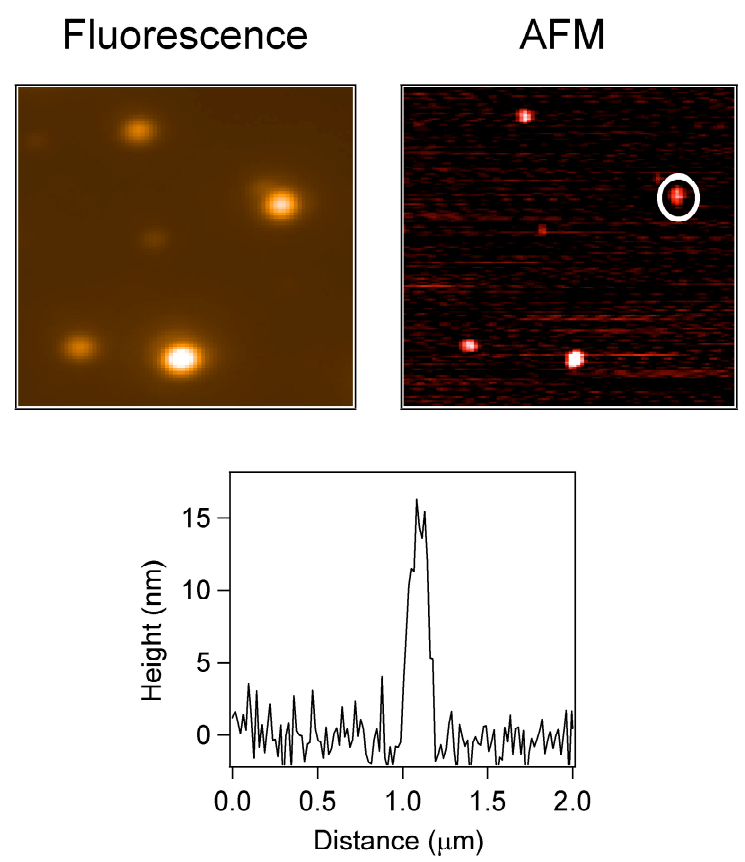

Figure 1 Fluorescence image (left) correlated with atomic force microscope image (right) of the same individual CdSeOPV nanostructures. The height trace of the circled nanostructure is also included (bottom). The real-space dimensions of the two images are $2.5 \times 2.5 \mu \mathrm{m}$. 


\section{Absorption and Photoluminescence Emission Curves}

Figure 2 shows the absorption curve for a $4.5 \mathrm{~nm}$ CdSe quantum dot sample along with a photoluminescence spectrum typical of the oligo-phenylene vinylene (OPV) ligands used in quantum dot-OPV nanostructure single-molecule experiments. The blue edge of the OPV photoluminescence spectrum of OPV is truncated by reflection of wavelengths $<500 \mathrm{~nm}$ from the dichroic filter used for 457-nm excitation (arrow).

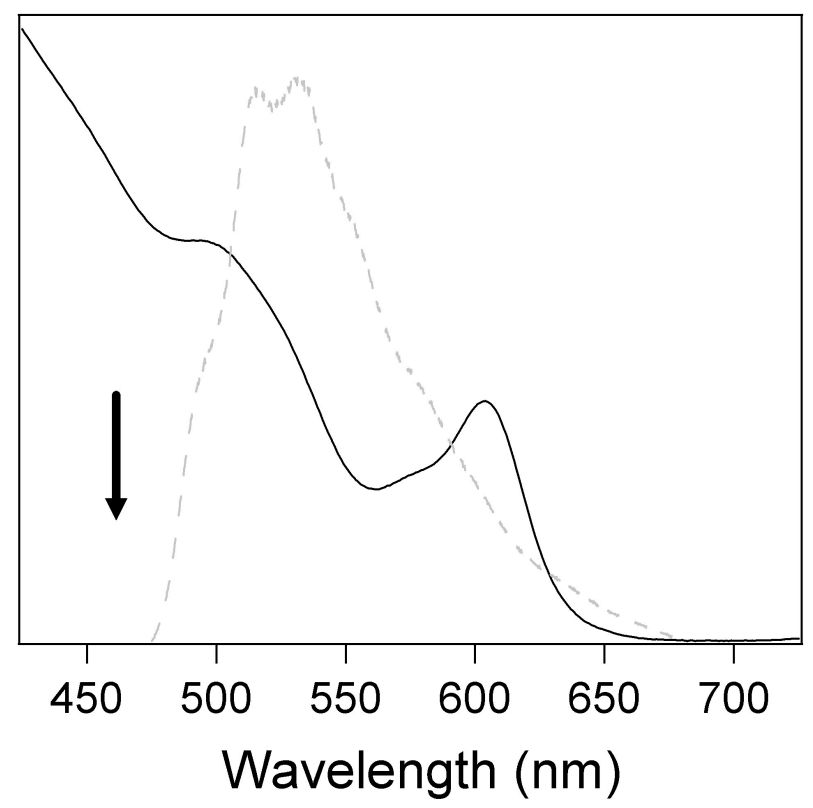

Figure 2 Absorption curve for $\sim 4.5 \mathrm{~nm}$ CdSe quantum dots (solid curve) and OPV photoluminescence spectrum (dashed curve). The arrow denotes the wavelength location of the excitation laser (457 $\mathrm{nm})$. 